\title{
Protective effect of polyphenol-rich extract prepared from Malaysian cocoa (Theobroma cacao) on glucose levels and lipid profiles in streptozotocin-induced diabetic rats.
}

\begin{abstract}
BACKGROUND: Cocoa beans are used for preparing cocoa liquor and cocoa powder, which are the main ingredients of cocoa-based products. Previous studies have reported the health benefits of cocoa polyphenols in reducing the risk of cardiovascular diseases. However, there is no report on the efficacy of cocoa polyphenols on diabetes mellitus. Therefore this study was designed to evaluate the protective effect of cocoa polyphenol-rich extract (CE) on glucose levels and lipid profiles in streptozotocin (STZ)-induced diabetic rats. Male SpragueDawley rats were divided into diabetic control, diabetic $\mathrm{CE}$ and diabetic glibenclamide groups. RESULTS: Three different dosages of CE (10, 20 and $30 \mathrm{mg}$ per $100 \mathrm{~g}$ body weight) were administered orally once a day for 1 week before STZ injection and for 3 weeks thereafter. The results showed that CE could normalise the body weight loss caused by STZ. In the $20 \mathrm{mg}$ CE-pretreated group there was a 143\% increase in plasma glucose levels, compared with a $226 \%$ increase in diabetic control rats. CE could also normalise total cholesterol, triglycerides and high-density lipoprotein cholesterol at the end of the experiment compared with the baseline. CONCLUSION: The present study suggests that pretreatment with CE from roasted cocoa beans could prevent the development of diabetes induced by STZ injection in rats.
\end{abstract}

Keyword: Theobroma cacao; Cocoa extract; Glucose levels; Hypoglycaemic. 